\title{
Perceptionization of FM/FD/1 queuing model under various fuzzy numbers
}

\author{
Usha Prameela Karupothu ${ }^{1, *}$ and Pavan Kumar ${ }^{1}$ \\ ${ }^{1}$ Koneru Lakshmaiah Education Foundation, Koneru Lakshmaiah University \\ Vaddeswaram, Guntur, AP-522502, India \\ E-mail: 〈\{kushaprameela, pavankmaths\}@gmail.com〉
}

\begin{abstract}
We present a FM/FD/1 queuing model with unbounded limit under different fuzzy numbers. The arrival (landing) rate and service (administration) rate are thought to be fuzzy numbers such as triangular, trapezoidal and pentagonal fuzzy numbers. Because random event can only be observed in an uncertain manner, the fuzzy result of an uncertainty mapping is a fuzzy random variable. Consequently, it is conceivable to characterize the specific connection between randomness and fuzziness. The execution proportions of this lining miniature are fuzzified after that examined by utilizing $\alpha$-cut estimations and DSW algorithm (Dong, Shah and Wong). Relating to different fuzzy numbers, the numerical precedents are delineated to test the attainability of this model (miniature). A comparative illustration corresponding to each fuzzy number is accomplished for various estimations of $\alpha$.
\end{abstract}

Keywords: DSW algorithm, fuzzy number, perceptionization, queuing model, $\alpha$ - cut

Received: April 09, 2020; accepted: June 16, 2020; available online: July 07, 2020

DOI: $10.17535 /$ crorr.2020.0011

\section{Introduction}

Queue contains of at least one queues or one or more overhauled offices under a set of guidelines. The parameters landing rate $(\lambda)$ and administration rate $(\mu)$ are required to pursue dissemination in queuing hypothesis. Kanufmann [12] in 1975 depicted introduction to the theory of fuzzy subsets. Gross et al. [21] in 1985 proposed a few basics of queuing hypothesis. Yager [29] in 1986 displayed a portrayal of the augmentation standard. Fuzzy queuing model was introduced by Lie et al. [15] in 1989. Negi et al. [17] in 1992 presented analysis of queuing systems. As of late, George et al. [10] in 1995 portrayed the fuzzy sets and logic. Chen [4, 5] in 2005 proposed a parametric nonlinear programming approach to fuzzy queues with mass administration and in 2006 presented an arithmetic programming way to deal with the machine obstruction issue with fuzzy parameters. He developed FM/FM/1/ $\infty / F C F S$, where FM means fuzzified exponential time dependent on queuing hypothesis. K. Gupta et al. [7] in 2007 discussed various queuing model in his book. Timothy J. Rose [24] in 2010 proposed a fuzzy logic with engineering applications. S. Barak et al. [3] in 2012 discussed the cost analysis of fuzzy queuing systems. Srinivasan [22] in 2014 presented a fuzzy queuing model utilizing DSW algorithm. Shanmugasundaram et al. [20] in 2015 proposed a miniature on fuzzy multi server queuing model through DSW calculation. The fuzzy set can be part into lighten patent points along $\alpha$-cut technique, additionally DSW algorithm is utilized to characterize an enrolment capacity of the execution measure in queuing models. With regards to customary lining model, both the parameters, that is, the inter landing times and administration times are required

${ }^{*}$ Corresponding author. 
to pursue certain appropriations. In common practice the landing rate, administration rate is much of the time portrayed by etymological terms, for example, high, low, extremely low and moderate can be best depicted by the fuzzy sets. A FM/FD/1 queue, the simplest queue with deterministic service time, has a variety of applications in the performance evaluation of production management, telecommunications networks, and other areas. The primary thought of this paper is to acquire the exact crisp values from the fuzzy values and then applying within the queuing performance formulas to 3 sorts of participation capacities, i.e. triangular, trapezoidal and pentagonal enrolment capacities. Fuzzy Queuing models are also studied by Mueen [16], Julia Rose Mary [11], Rakesh Kumar [19], Vasanth Kumar [9, 27], Usha Prameela and Pavan [25, 26], Aria [1, 2], Wagner [28] Hajipour [8], Fazzolari [6], Kobayashi [13], Kumar [14], Sushil [23] and Novak [18]. In Section 2, some basic ideas and definitions are presented. In Section 3, the presumptions and notations are described. In Section 4 the proposed queuing miniature is given. In Section 5, the result approach to the present model is described. In Section 6, three numerical precedents are solved. In Section 7 the results and discussions are presented. In Section 8 the model is concluded.

\section{Basic definitions}

\subsection{Fuzzy number}

A fuzzy set $\tilde{A}$ is characterized on the set of real numbers $R$ is said to be fuzzy number if it has the accompanying qualities such as $\tilde{A}$ is normal, convex and the support of it is closed and bounded [12]

\section{2. $\alpha-$ cut}

An $\alpha$-cut of a fuzzy set [12] is a crisp set $A$ that contains all the elements of the universal set $X$ that have a participation grade in $\tilde{A}$ greater than or equal to determined estimation of $\alpha$, thus

$$
\alpha=\left\{x \in X: \mu_{\tilde{A}}(x) \geq \alpha, 0 \leq \alpha \leq 1\right\} .
$$

\subsection{Arithmetic for interval analysis}

Let the two interval numbers designated by ordered pairs of real numbers with lower and upper limits be $G=\left[a_{1}, a_{2}\right], a_{1} \leq a_{2}$ and $H=\left[b_{1}, b_{2}\right], b_{1} \leq b_{2}$, with following properties:

$$
\begin{aligned}
& {\left[a_{1}, a_{2}\right]+\left[b_{1}, b_{2}\right]=\left[a_{1}+b_{1}, a_{2}+b_{2}\right]} \\
& {\left[a_{1}, a_{2}\right]-\left[b_{1}, b_{2}\right]=\left[a_{1}-b_{1}, a_{2}-b_{2}\right]} \\
& {\left[a_{1}, a_{2}\right] \times\left[b_{1}, b_{2}\right]=\left[\min \left(a_{1} b_{1}, a_{1} b_{2}, a_{2} b_{1}, a_{2} b_{2}\right), \max \left(a_{1} b_{1}, a_{1} b_{2}, a_{2} b_{1}, a_{2} b_{2}\right)\right]} \\
& {\left[a_{1}, a_{2}\right] \div\left[b_{1}, b_{2}\right]=\left[a_{1}, a_{2}\right] \times\left[1 / b_{1}, 1 / b_{2}\right] \text { provided that } 0 \text { does not belong to }\left[b_{1}, b_{2}\right]} \\
& \alpha \times\left[a_{1}, a_{2}\right]=\left[\alpha a_{1}, \alpha a_{2}\right] \text { for } \alpha>0 \text { and }\left[\alpha a_{2}, \alpha a_{1}\right] \text { for } \alpha<0 .
\end{aligned}
$$

\section{Presumptions and notations}

In the present model, accompanying presumptions are utilized similar as in [13]:

i) Unbounded limit of $\mathrm{FM} / \mathrm{FD} / 1 / \infty / \mathrm{FCFS}$ queuing model with one server

ii) Exponentially disseminated arrival times 
iii) Deterministic service appropriation, i.e. fixed

iv) Landing rate, administration rate are fuzzy numbers.

Notations are given as:

$$
\begin{aligned}
\mu & =\text { average number of clients being overhauled per unit of time } \\
\lambda & =\text { average number of clients arriving per unit of time } \\
L_{s} & =\text { average number of clients in the framework } \\
L_{q} & =\text { average number of clients holding up in the line } \\
W_{s} & =\text { average holding up time of a client in the framework } \\
W_{q} & =\text { average holding up time of a client in the line } \\
X & =\text { set of the inter entry time } \\
Y & =\text { set of the administration time } \\
A & =\text { inter entry time } \\
S & =\text { administration times }
\end{aligned}
$$

\section{Proposed queuing model}

We propose a single server queuing model, with first come first served (FCFS) regulation [13, 18]. It is indicated in Kendall's notation as (FM/FD/1):( $\infty / F C F S)$. Here FM denotes fuzzified exponential dispersion with landing rate $\lambda$ and FD denotes fuzzified steady (constant) dispersion with administration rate $\mu$. This process is stochastic whose state space is the set $\{0,1,2,3, \ldots\}$ where the value indicates the number of customers present in the system, incorporating any entity at present in administration. It is of infinite size, so there is no restriction on the number of customers it can contain. The execution proportions of the proposed model are given as similar to [23]. Namely, expected range of consumers within the system is:

$$
L_{s}=\rho+\frac{\rho^{2}}{2(1-\rho)}, \quad \rho=\frac{\lambda}{\mu} .
$$

The expected range of consumers within the queue is:

$$
L_{q}=\frac{\rho^{2}}{2(1-\rho)} .
$$

The expected time a client spends within the queue is:

$$
W_{q}=\frac{\rho}{2(1-\rho) \mu} .
$$

The expected time a client spends within the system is:

$$
W_{s}=\frac{1}{\mu}+\frac{\rho}{2(1-\rho) \mu} .
$$




\section{Solution procedure}

DSW (Dong, Shah and Wong) is a rough technique utilizes intervals at various $\alpha$-cut dimensions in characterizing execution proportions [15]. It avoids variation from the output membership function because of use of the segregation reaching on the fuzzy variable area. Any persistent participation capacity can be spoken to by ceaseless scope of $\alpha$-cut in term from $\alpha=0$ to $\alpha=1$. Let $\mu_{\tilde{A}}(a)$ and $\mu_{\hat{S}}(s)$ be membership functions of the inter entry time and the overhauled time, separately. The inter arrival time and service times are fuzzy sets, depicted as:

$$
\tilde{A}=\left\{\left(a, \mu_{\tilde{A}}(a)\right), a \in X\right\} \text { and } \hat{S}=\left\{\left(s, \mu_{\hat{S}}(s)\right), \quad s \in Y\right\} .
$$

The $\alpha$-cuts of inter entry time, overhauled time are represented as:

$$
\tilde{A}(\alpha)=\left\{\mu_{\tilde{A}}(a) \geq 0, \quad a \in X\right\} \text { and } \hat{S}(\alpha)=\left\{\mu_{\hat{S}}(s) \geq a, \quad s \in Y\right\} .
$$

The DSW calculation contains the accompanying steps:

Step 1. Stipulate $\alpha$-cut esteem where $0 \leq \alpha \leq 1$

Step 2. Discover the intervals in the input membership functions that compare to this $\alpha$

Step 3. Utilizing standard binary interval operations, process the interval for the output membership function for the chosen $\alpha$-cut dimension

Step 4. Iterate stages 1-3 for various estimations of $\alpha$ to finish $\alpha$-cut portrayal of the arrangement

\section{Numerical precedents}

We explain the accompanying numerical models, thinking about different fuzzy numbers.

\subsection{Precedent 1}

Consider an FM/FD/1/ $\infty /$ FIFO model where both the entry rate and overhauled rate are triangular fuzzy numbers represented by $\lambda=[1,2,3]$ and $\mu=[11,12,13]$. The interval of certainty at probability level $\alpha$ as $[1+\alpha, 3-\alpha]$ and $[11+\alpha, 13-\alpha]$. By taking $\alpha$ values from $0,0.1, \ldots, 1$ the execution measures are shown in the Table 1.

\begin{tabular}{|c|cccc|}
\hline$\alpha$ & $L_{q}$ & $L_{s}$ & $W_{s}$ & $W_{q}$ \\
\hline \hline 0 & {$[0.0032,0.0511]$} & {$[0.0801,0.3238]$} & {$[0.0801,0.1079]$} & {$[0.0032,0.0170]$} \\
0.1 & {$[0.0039,0.0461]$} & {$[0.0892,0.3074]$} & {$[0.0811,0.1060]$} & {$[0.0036,0.0159]$} \\
0.2 & {$[0.0048,0.0416]$} & {$[0.0985,0.2916]$} & {$[0.0821,0.1041]$} & {$[0.0040,0.0148]$} \\
0.3 & {$[0.0058,0.0375]$} & {$[0.1082,0.2764]$} & {$[0.0832,0.1023]$} & {$[0.0044,0.0138]$} \\
0.4 & {$[0.0069,0.0336]$} & {$[0.1180,0.2617]$} & {$[0.0843,0.1006]$} & {$[0.0049,0.0129]$} \\
0.5 & {$[0.0081,0.0301]$} & {$[0.1281,0.2475]$} & {$[0.0854,0.0990]$} & {$[0.0054,0.0120]$} \\
0.6 & {$[0.0095,0.0269]$} & {$[0.1385,0.2338]$} & {$[0.0866,0.0974]$} & {$[0.0059,0.0112]$} \\
0.7 & {$[0.0110,0.0240]$} & {$[0.1492,0.2202]$} & {$[0.0878,0.0959]$} & {$[0.0065,0.0104]$} \\
0.8 & {$[0.0127,0.0213]$} & {$[0.1603,0.2078]$} & {$[0.0890,0.0944]$} & {$[0.0070,0.0097]$} \\
0.9 & {$[0.0146,0.0189]$} & {$[0.1716,0.1953]$} & {$[0.0903,0.0930]$} & {$[0.0076,0.0090]$} \\
1 & {$[0.0166,0.0166]$} & {$[0.1833,0.1833]$} & {$[0.0916,0.0916]$} & {$[0.0083,0.0083]$} \\
\hline
\end{tabular}

Table 1: The $\alpha$-cuts of $L_{s}, L_{q}, W_{s}$ and $W_{q}$ at $\alpha$ values (precedent 1) 
Perceptionization of FM/FD/1 queuing model under various fuzzy numbers

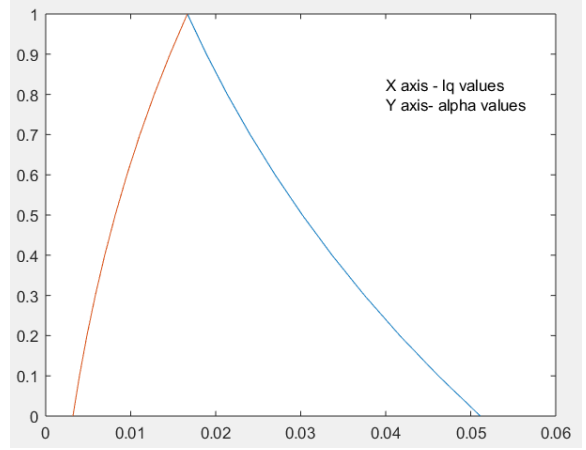

(a) $L_{q}$

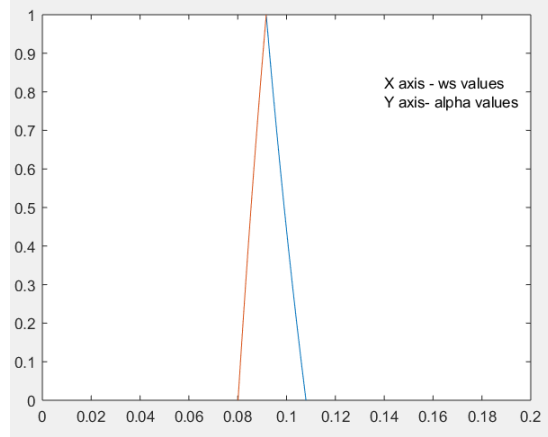

(c) $W_{s}$

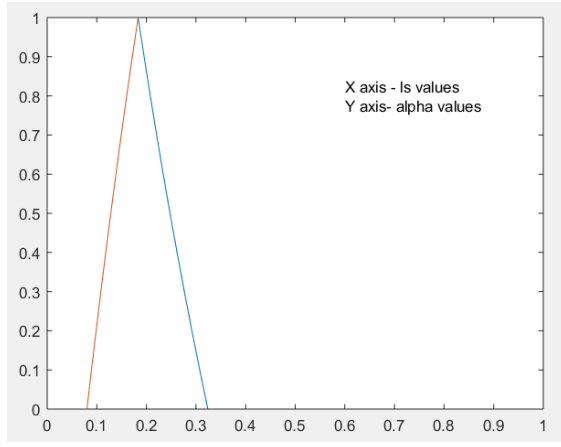

(b) $L_{s}$

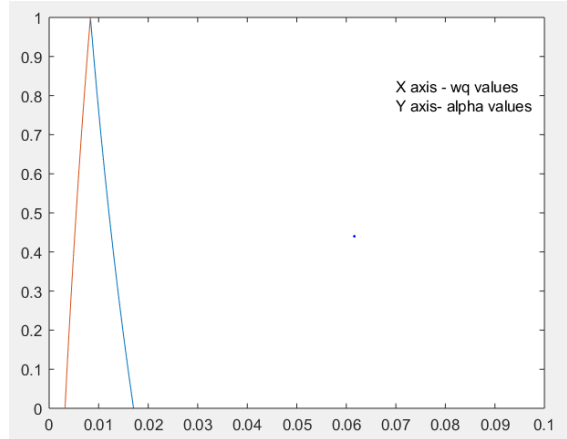

(d) $W_{q}$

Figure 1: Graphs of the $L_{q}, L_{s}, W_{s}$ and $W_{q}$ (precedent 1)

\subsection{Precedent 2}

Acknowledge that both landing rate and administration rate are trapezoidal fuzzy numbers represented by $\lambda=[1,2,3,4]$ and $\mu=[11,12,13,14]$. The interval of certainty at probability level $\alpha$ as $[1+\alpha, 4-\alpha]$ and $[11+\alpha, 14-\alpha]$, where $x=[1+\alpha, 4-\alpha]$ and $y=[11+\alpha, 14-\alpha]$. By taking $\alpha$ values from $0,0.1, \ldots, 1$ the execution measures are shown in the Table 2.

\begin{tabular}{|c|cccc|}
\hline$\alpha$ & $L_{q}$ & $L_{s}$ & $W_{s}$ & $W_{q}$ \\
\hline \hline 0 & {$[0.0027,0.1039]$} & {$[0.0741,0.4675]$} & {$[0.0741,0.1168]$} & {$[0.0027,0.0259]$} \\
0.1 & {$[0.0034,0.0951]$} & {$[0.0825,0.4465]$} & {$[0.0750,0.1144]$} & {$[0.0030,0.0243]$} \\
0.2 & {$[0.0041,0.0871]$} & {$[0.0910,0.4264]$} & {$[0.0759,0.1122]$} & {$[0.0034,0.0229]$} \\
0.3 & {$[0.0049,0.0797]$} & {$[0.0998,0.4071]$} & {$[0.0768,0.1100]$} & {$[0.0038,0.0215]$} \\
0.4 & {$[0.0059,0.0728]$} & {$[0.1088,0.3886]$} & {$[0.0777,0.1079]$} & {$[0.0042,0.0202]$} \\
0.5 & {$[0.0069,0.0665]$} & {$[0.1180,0.3709]$} & {$[0.0787,0.1059]$} & {$[0.0046,0.0190]$} \\
0.6 & {$[0.0080,0.0607]$} & {$[0.1275,0.3538]$} & {$[0.0796,0.1040]$} & {$[0.0050,0.0178]$} \\
0.7 & {$[0.0093,0.0554]$} & {$[0.1371,0.3374]$} & {$[0.0806,0.1022]$} & {$[0.0055,0.0167]$} \\
0.8 & {$[0.0107,0.0504]$} & {$[0.1471,0.3216]$} & {$[0.0817,0.1005]$} & {$[0.0059,0.0157]$} \\
0.9 & {$[0.0123,0.0458]$} & {$[0.1573,0.3063]$} & {$[0.0828,0.0988]$} & {$[0.0064,0.0148]$} \\
1 & {$[0.0139,0.0416]$} & {$[0.1678,0.2916]$} & {$[0.0839,0.0972]$} & {$[0.0069,0.0138]$} \\
\hline
\end{tabular}

Table 2: The $\alpha$-cuts of $L_{s}, L_{q}, W_{s}$ and $W_{q}$ at $\alpha$ values (precedent 2) 


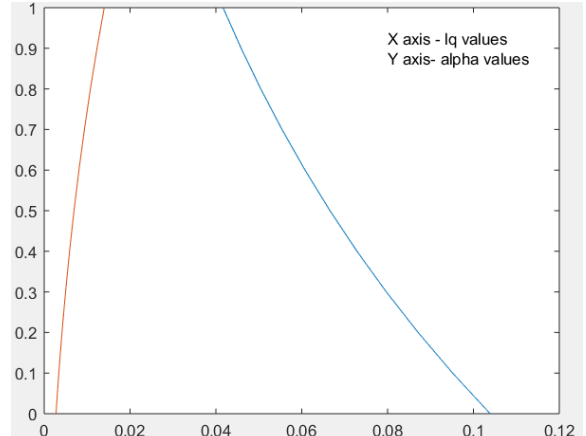

(a) $L_{q}$

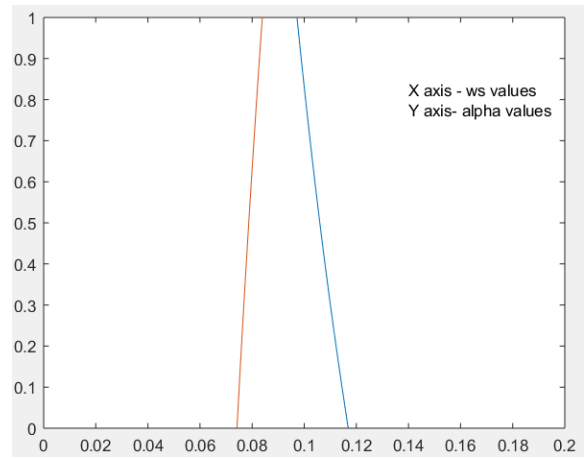

(c) $W_{s}$

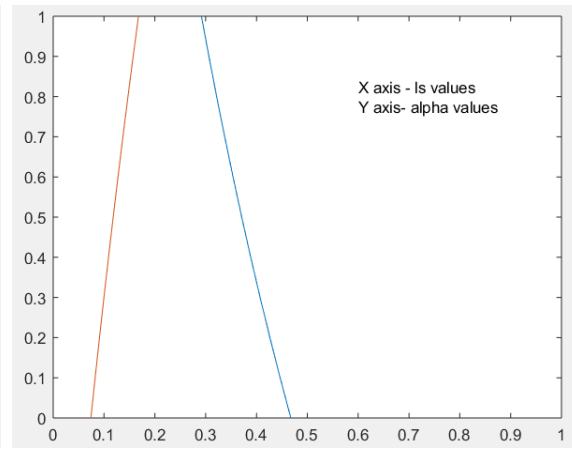

(b) $L_{s}$

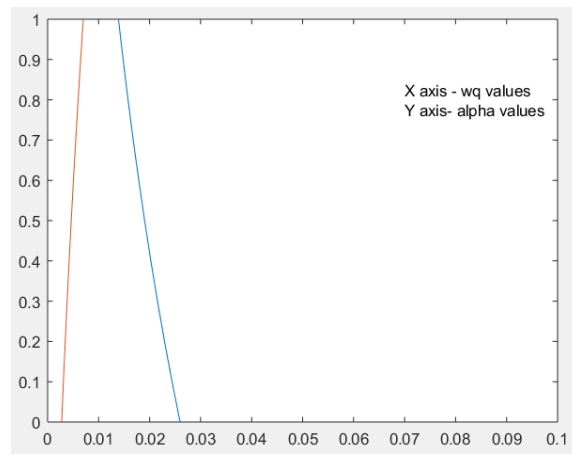

(d) $W_{q}$

Figure 2: Graphs of the $L_{q}, L_{s}, W_{s}$ and $W_{q}$ (precedent 2)

\subsection{Precedent 3}

Recognize both the entry and overhauled rate are pentagonal fuzzy numbers represented by $\lambda=[1,2,3,4,5]$ and $\mu=[11,12,13,14,15]$. The interval of certainty at probability level $\alpha$ as $[1+\alpha, 5-\alpha]$ and $[11+\alpha, 15-\alpha]$, where $x=[1+\alpha, 5-\alpha]$ and $y=[11+\alpha, 15-\alpha]$. By taking $\alpha$ values from $0,0.1, \ldots, 1$ the execution measures are shown in the Table 3.

\begin{tabular}{|c|cccc|}
\hline$\alpha$ & $L_{q}$ & $L_{s}$ & $W_{s}$ & $W_{q}$ \\
\hline \hline 0 & {$[0.0023,0.1893]$} & {$[0.0690,0.6439]$} & {$[0.0690,0.1287]$} & {$[0.0023,0.0378]$} \\
0.1 & {$[0.0035,0.1607]$} & {$[0.0846,0.5892]$} & {$[0.0705,0.1227]$} & {$[0.0029,0.0334]$} \\
0.2 & {$[0.0050,0.1364]$} & {$[0.1009,0.5399]$} & {$[0.0721,0.1173]$} & {$[0.0036,0.0296]$} \\
0.3 & {$[0.0069,0.1159]$} & {$[0.1180,0.4952]$} & {$[0.0737,0.1125]$} & {$[0.0043,0.0263]$} \\
0.4 & {$[0.0092,0.0983]$} & {$[0.1359,0.4542]$} & {$[0.0755,0.1081]$} & {$[0.0051,0.0234]$} \\
0.5 & {$[0.0119,0.0833]$} & {$[0.1547,0.4166]$} & {$[0.0773,0.1041]$} & {$[0.0059,0.0208]$} \\
0.6 & {$[0.0151,0.0704]$} & {$[0.1745,0.3819]$} & {$[0.0793,0.1005]$} & {$[0.0068,0.0185]$} \\
0.7 & {$[0.0189,0.0593]$} & {$[0.1953,0.3497]$} & {$[0.0814,0.0971]$} & {$[0.0078,0.0164]$} \\
0.8 & {$[0.0233,0.0498]$} & {$[0.2173,0.3197]$} & {$[0.0836,0.0940]$} & {$[0.0089,0.0146]$} \\
0.9 & {$[0.0285,0.0416]$} & {$[0.2406,0.2916]$} & {$[0.0859,0.0911]$} & {$[0.0101,0.0130]$} \\
1 & {$[0.0346,0.0346]$} & {$[0.2653,0.2653]$} & {$[0.0884,0.0884]$} & {$[0.0115,0.0115]$} \\
\hline
\end{tabular}

Table 3: The $\alpha$-cuts of $L_{s}, L_{q}, W_{s}$ and $W_{q}$ at $\alpha$ values (precedent 3) 


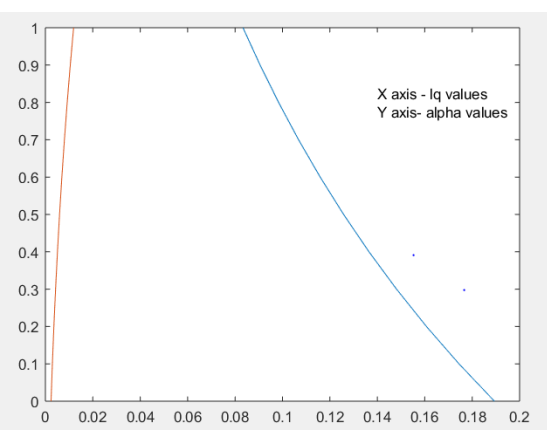

(a) $L_{q}$

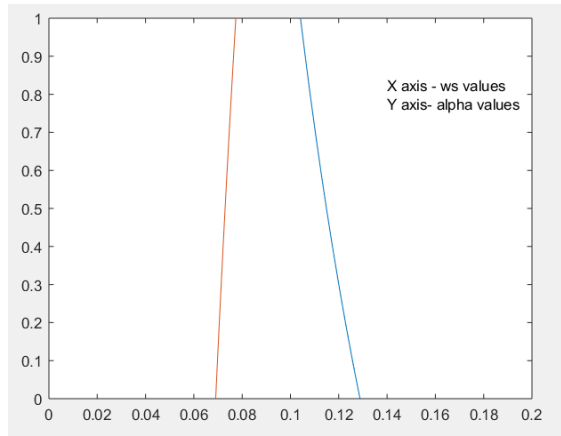

(c) $W_{s}$

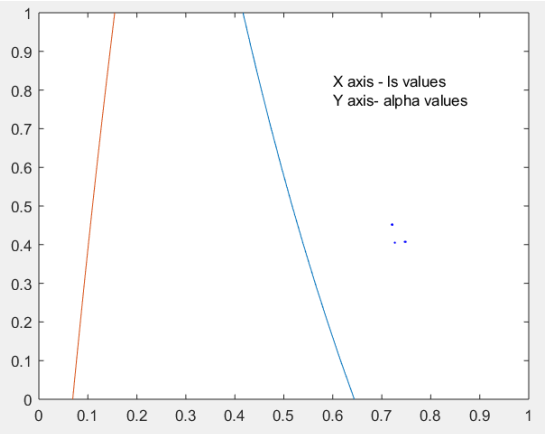

(b) $L_{s}$

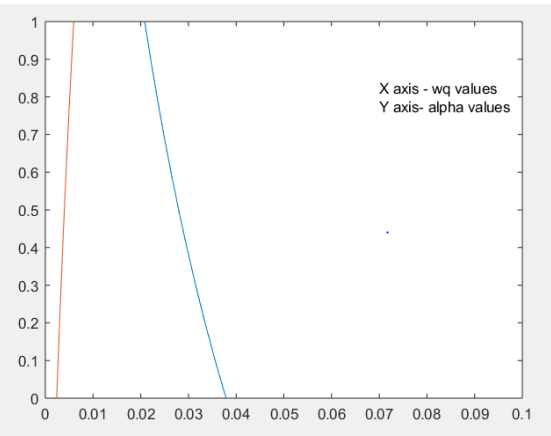

(d) $W_{q}$

Figure 3: Graphs of the $L_{q}, L_{s}, W_{s}$ and $W_{q}$ (precedent 3)

\section{Results and discussions}

Utilizing MATLAB, we achieve $\alpha$-cuts of entry rate, administration rate and fuzzy anticipated number of occupations in queue just as system at eleven patent dimensions: $0,0.1,0.2,0.3$, $0.4,0.5,0.6,0.7,0.8,0.9$ and 1 . Crisp intervals for fuzzy anticipated number of employments in queue and system at various probability $\alpha$ levels are exhibited in Tables 1-3. The execution proportions for example anticipated number of clients in the system $L_{a}$, anticipated length of the line $L_{q}$, the average holding up time of a client in the system $W_{s}$ and the average holding up time of a client in the queue $W_{q}$ likewise inferred in Tables 1-3.

From the Table 1 we can extract following information:

i) Anticipated number of clients in the line is 0.0166 and inconceivable falls outside $[0.0032$, 0.0511]

ii) Anticipated number of clients in the system is 0.1833 and inconceivable falls outside [0.0801, 0.3238]

iii) Average holding up time of a client in the line is 0.0083 and inconceivable falls outside $[0.0032,0.0170]$

iv) Average holding up time of a client in the system is 0.0916 and inconceivable falls outside [0.0801, 0.1079] 
From the Table 2 we can extract following information:

v) Expected number of clients in the line is 0.0416 and inconceivable falls outside $[0.0027$, 0.1039]

vi) Anticipated number of clients in the framework is 0.2916 and inconceivable falls outside $[0.0741,0.4675]$

vii) Average holding up time of a client in the queue is 0.0138 and inconceivable falls outside $[0.0027,0.0259]$

viii) Average holding up time of a client in the system is 0.0972and inconceivable falls outside [0.0741, 0.1168]

From the Table 3 we can extract following information:

ix) Anticipated number of clients in the line is 0.0346 and inconceivable falls outside [0.0023, 0.1893]

$\mathrm{x})$ Anticipated number of clients in the framework is 0.2653 and inconceivable falls outside [0.0690, 0.6439]

xi) Average holding up time of a client in the line is 0.0115 and inconceivable falls outside $[0.0023,0.0378]$

xii) Average holding up time of a customer in the system is 0.0884 and inconceivable falls outside $[0.0690,0.1287]$

Finaly, comparison of FM/FD/1 queuing model without fuzzy number and the FM/FD/1 queuing model with various fuzzy numbers is given in Table 4.

\begin{tabular}{|c|c|c|c|c|}
\hline & FM/FD/1 & \multicolumn{3}{|c|}{ FM/FD/1 with fuzzy number } \\
\cline { 3 - 5 } & $\begin{array}{c}\text { without } \\
\text { fuzzy number } \\
\end{array}$ & $\begin{array}{c}\text { Triangular fuzzy } \\
\text { number } \lambda=[1,2,3]\end{array}$ & $\begin{array}{c}\text { Trapezoidal fuzzy } \\
\text { number } \lambda=[1,2,3,4]\end{array}$ & $\begin{array}{c}\text { Pentagonal fuzzy } \\
\text { number } \lambda=[1,2,3,4,5] \\
\mu=[1,12,13,14,15]\end{array}$ \\
\hline \hline$L_{q}$ & 0.0166 & {$[0.0032,0.0511]$} & {$[0.0027,0.1039]$} & {$[0.0023,0.1893]$} \\
$L_{s}$ & 0.1832 & {$[0.0801,0.3238]$} & {$[0.0741,0.4675]$} & {$[0.0690,0.6439]$} \\
$W_{s}$ & 0.0916 & {$[0.0801,0.1079]$} & {$[0.0741,0.1168]$} & {$[0.0690,0.1287]$} \\
$W_{q}$ & 0.0083 & {$[0.0032,0.0170]$} & {$[0.0027,0.0259]$} & {$[0.0023,0.0378]$} \\
\hline
\end{tabular}

Table 4: Comparison of FM/FD/1 queuing model without and with fuzzy number

In this investigation, the outcome shows that the exhibition measures $L_{q}, L_{s}, W_{s}$ and $W_{q}$ for both queuing theory model and fuzzy queuing model were processed and analysed. In view of the outcome, the fuzzy queuing model is substantially more successful and proficient to measure the exhibition of $\mathrm{FM} / \mathrm{FD} / 1$ lining framework since the fuzzy set hypothesis is more effectively versatile. Applying the fuzzy lining model gives more extensive data, which will be exceptionally valuable in characterizing a lining framework. Hence, this investigation infers that fuzzy lining is one of the elective approaches to register the exhibition measures since the data acquired from the application is a lot clearer to understand and interpret. 


\section{Conclusion}

Here, we surmise that fuzzy set hypothesis has been connected to lining hypothesis more over the inter landing time and administration time are fuzzy identity. The execution proportions for example entity length, line length, entity time, line time and so forth are likewise fuzzy in nature. Numerical precedents demonstrate the proficiency of DSW calculation. Here it is noticed that the achievement of queuing model can be upgraded by expanding the number of variables. The proposed model can help the businesses, wholesalers and retailers in precisely deciding the ideal execution of the queuing system. There are different viewpoints that the paper can be extended. One of them is to think about random variable, or fuzzy random variable to landing rate and administration rate. Another conceivable dimensional to stretch out this paper is to consider intuitionistic fuzzy numbers

\section{References}

[1] Aria, M. (2011). Intelligent traffic light control based on interval type 2 fuzzy system. Journal Majalah Ilmiah Unikom, 8(2), 203-216. https://jurnal.unikom.ac.id/jurnal/ intelligent-traffic-light.1p

[2] Aria, M. (2019). New fuzzy logic system for controlling multiple traffic intersections with dynamic phase selection and pedestrian crossing signal. Journal of Engineering, Science and Technology, 14(4), 1974-1983. http://jestec.taylors.edu.my/Vol $\% 2014 \% 20$ issue $\% 204 \% 20$ August $\%$ 202019/14_4_16.pdf

[3] Barak, S. and Fallahnezhad, M. S. (2012). Cost analysis of fuzzy queuing systems. International Journal of Applied Operational Research, 2(2), 25-36. http://ijorlu.liau.ac.ir/ article-1-138-en.pdf

[4] Chen, S. P. (2005). Parametric nonlinear programming approach to fuzzy queues with bulk service. European Journal of Operational Research, 163(2), 434-444. doi: 10.1016/j.ejor.2003.10.041

[5] Chen, S. P. (2006). A mathematical programming approach to the machine interference problem with fuzzy parameters. Applied Mathematics and Computation, 174(1), 374-387. doi: 10.1016/j.amc.2005.05.012

[6] Fazzolari, M., Alcala, R. Nojima, Y. Ishibuchi, H and Herrera,(2013). A review of the application of multi objective evolutionary fuzzy systems: current status and further Directions. IEEE Transactions on Fuzzy Systems, 21(1), 45-65. doi: 10.1109/tfuzz.2012.2201338

[7] Gupta, P. K. and Hira, D.S. (2007). Operations Research, 884-885.

[8] Hajipour, V., Khodakarami, V. and Tavana, M. (2014). The redundancy queuing -locationallocation problem: A novel approach. IEEE Transactions on Engineering Management, 61(3), 534-544. doi: 10.1109/tem.2014.2317760

[9] Hanumantha Rao, S., Vasanta Kumar, V., Srinivasa Rao, T. and Srinivasa Kumar, B. (2018). Analysis of batch arrival two-phase $\mathrm{Mx} / \mathrm{M} / 1$ queuing system with impatient customers and unreliable server. Journal of Advanced Research in Dynamical and Control Systems, 10(2), 348-356. https : //www . jardcs . org/backissues/abstract . php? archiveid=2933

[10] Lofti, A. Z. (1996). Fuzzy sets, fuzzy logic and fuzzy systems. In: Advances in Fuzzy SystemsApplications and Theory, George J Klir and Bo Yuan (Eds.) doi: 10.1142/2895

[11] Julia Rose Mary, K. and Manjula Christina, (2015). Analysis of total average cost of $M_{m, N}^{X} / M / 1 / B D / M V$ with fuzzy parameters using robust ranking technique. International Journal of Computer Applications, 121(24), 1-4. doi: 10.5120/21870-4362

[12] Kanufmann, A. (1975). Introduction to the theory of fuzzy subsets, Vol.I. Academic Press, New York.

[13] Kobayashi, H. and Konheim, A. (1977). Queueing models for computer communications system analysis. IEEE Transactions on Communications, 25(1), 2-29. doi: 10.1109/tcom.1977.1093702

[14] Kumar, P. (2019). An inventory planning problem for time-varying linear demand and parabolic holding cost with salvage value. Croatian Operational Research Review, 10(2), 187-199. doi: 10.17535 /crorr. 2019.0017 
[15] Li, R. J and Lee, E. S. (1989). Analysis of fuzzy queues. Computers and Mathematics with Applications, 17(7), 1143-1147. doi: 10.1016/0898-1221(89)90044-8

[16] Mueen, Z. Ramli, R. Zabidi, N. Z. (2016). Analysis of performance measures with single channel fuzzy queues under two class by ranking method. AIP Conference Proceedings, 1761(1), 20-77. doi: $10.1063 / 1.4960917$

[17] Negi, D. S. and Lee, E. S. (1992). Analysis and simulation of fuzzy queues. Fuzzy Sets and Systems, 46(3), 321-330. doi: 10.1016/0165-0114(92)90370-j

[18] Novak, and Watson, R., (2009). Determining an adequate probe separation for estimating the arrival rate in an M/D/1 queue using single-packet probing. Queueing Systems, 61(4), 255-272. doi: $10.1007 / \mathrm{s} 11134-009-9107-\mathrm{z}$

[19] Rakesh Kumar, and Bhupender Kumar Som, (2015). A finite single server queuing system with reverse reneging. American Journal of Operational Research, 5(5), 125-128. doi: 10.5923/j.ajor.20150505.03

[20] Shanmugasundaram, S. and Venkatesh, B. B. (2015). Study of fuzzy multi server queuing model through DSW algorithm.International Journal of Latest Trends in Engineering and Technology, 5(3), 452-457. https://www.ijltet.org/journal_details.php?id=884\&j_id=2012

[21] Shortle, J. F., Thompson, J. M., Gross, D. and Harris, C. M. (2018). Fundamentals of queuing theory (Wiley Series in Probability and Statistics). New York: John Wiley \& Sons, Inc. doi: $10.1002 / 9781119453765$

[22] Srinivasan, R. (2014). Fuzzy queueing model using DSW algorithm. International Journal of Advanced Research in Mathematics and Applications, 1(1), 57-62. http: //www . isrjournals.org/ journal-view/fuzzy-queueing-model-using-dsw-algorithm

[23] Sushil, G., Gyan Bahadur, T. Ram Prasad, G. and Sergei, S. (2017). A Survey on Queueing Systems with Mathematical Models and Applications. American Journal of Operational Research, 7(1), 1-14. doi: 10.5923/j.ajor.20170701.01

[24] Timothy, J. R. (2010). Fuzzy Logic with Engineering Applications (3rd Ed). John Wiley and Sons, Ltd. doi: 10.1002/9781119994374

[25] Usha Prameela, K.and Pavan Kumar, (2019). Execution proportions of multi server queuing model with pentagonal fuzzy number: DSW algorithm approach. International Journal of Innovative Technology and Exploring Engineering, 8(7), 1047-1051. https://www.ijitee.org/wp-content/ uploads/papers/v8i7/G5654058719.pdf

[26] Usha Prameela, K. and Pavan Kumar, (2019). FM/FEk/1 queuing model with Erlang service under various types of fuzzy numbers. International Journal of Recent Technology and Engineering, 8(1), 942-946. https://www.ijrte.org/wp-content/uploads/papers/v8i1/A1085058119.pdf

[27] Vasanta Kumar, V. and Chandan, K. (2008). Cost Analysis of two-phase $M X / E K / 1$ queuing system with N-policy. Opserach, 45(2), 155-174. doi: 10.1007/bf03398811

[28] Wagner, C. and Hagras, H. (2010). Uncertainty and type-2 fuzzy sets and systems. UK Workshop on Computational Intelligence (UKCI). Proceedings of IEEE publisher, Colchester, 1-5. doi: 10.1109/ukci.2010.5625603

[29] Yager, R. R. (1986). A characterization of the extension principle. Fuzzy Sets and Systems, 18(3), 71-78. doi: 10.1016/0165-0114(86)90002-3 